\title{
An alternative approach to determine attainable resolution directly from HREM images
}

\author{
A. Wang*, S. Turner, S. Van Aert, D. Van Dyck \\ Electron Microscopy for Materials Research (EMAT), University of Antwerp, Department of Physics, Groenenborgerlaan 171, 2020 Antwerp, Belgium
}

\section{A R T I C L E I N F O}

\section{Article history:}

Received 19 February 2013

Received in revised form

7 May 2013

Accepted 9 May 2013

Available online 23 May 2013

\section{Keywords:}

Resolution

Marr-Hildreth operator

Image processing

High-resolution electron microscopy

Channelling

\begin{abstract}
A B S T R A C T
The concept of resolution in high-resolution electron microscopy (HREM) is the power to resolve neighboring atoms. Since the resolution is related to the width of the point spread function of the microscope, it could in principle be determined from the image of a point object. However, in electron microscopy there are no ideal point objects. The smallest object is an individual atom. If the width of an atom is much smaller than the resolution of the microscope, this atom can still be considered as a point object. As the resolution of the microscope enters the sub-Å regime, information about the microscope is strongly entangled with the information about the atoms in HREM images. Therefore, we need to find an alternative method to determine the resolution in an object-independent way. In this work we propose to use the image wave of a crystalline object in zone axis orientation. Under this condition, the atoms of a column act as small lenses so that the electron beam channels through the atom column periodically. Because of this focusing, the image wave of the column can be much more peaked than the constituting atoms and can thus be a much more sensitive probe to measure the resolution. Our approach is to use the peakiness of the image wave of the atom column to determine the resolution. We will show that the resolution can be directly linked to the total curvature of the atom column wave. Moreover, we can then directly obtain the resolution of the microscope given that the contribution from the object is known, which is related to the bounding energy of the atom. The method is applied on an experimental $\mathrm{CaTiO}_{3}$ image wave.
\end{abstract}

(c) 2013 Elsevier B.V. All rights reserved.

\section{Introduction}

Originally the concept of resolution was defined by Lord Rayleigh [1] as the power to discriminate point objects, such as stars, with a telescope. In a sense, the resolution is related to the width of the point spread function of the telescope or the microscope. In [2,3], a review of various resolution definitions is given according to the transfer function in HREM. The most commonly used definition is the inverse of the information limit of the microscope $[2,4]$, which is determined by the damping envelope incorporating effects of partial coherence. In electron microscopy, the smallest objects are atoms and because the electron interacts with their electrostatic potential, the atoms cannot be considered as ideal point objects. This poses no problem when the resolution of the instrument is much larger than the width of the atom as was the case in the past. In that case, one can determine the resolution from the diffractogram of an amorphous thin film which can, to some extent, be considered as a white noise object $[5,6]$. Nowadays, with advanced techniques and aberration

\footnotetext{
* Corresponding author. Tel.: +32 3 2653252; fax: +32 32653318.

E-mail address: amy.wang@ua.ac.be (A. Wang).
}

correctors [7], the resolution of the microscope has been greatly improved to the sub-Å regime [8-11]. In this case, objects can no longer be considered as weak phase objects and dynamical scattering may become important, meaning the non-linear interaction may not be ignored [12]. The information about the microscope such as the resolution is evidently strongly entangled with the information about the atoms in the HREM images. Thus, the resolution cannot be defined independently of the object. Therefore, we must try to find an alternative method to determine the resolution in the image in an object-independent way. This means that the resolution in an image should be measured directly using the object under study.

In this work we propose to use the image wave of a crystal in zone axis orientation. In such a "channeling condition" [13] the atoms in a column act as small lenses that focus the electron wave. In this way the image wave amplitude can be much more sharply peaked than the width of the electrostatic potential of a single atom and this channeling occurs for light atoms as well as for heavy atoms. Furthermore, it has been shown in [15] that to a good approximation the image wave at the atom column position has a Gaussian shape. Our approach here is to use the shape of the image wave of an atom column in a zone axis condition to determine the resolution. 
The outline of this paper is as follows. In Section 2, an overview of the channelling theory is given. Next, in Section 3, the approach to determine the attainable resolution is derived. In Section 4, the influence of microscope lens aberrations on the attainable resolution is studied. Then, in Section 5, a practical example is given. Finally, in Section 6, conclusions are drawn.

\section{The channelling theory}

Due to the strong electrostatic potential of the atoms, an atom column in the direction of the electron beam acts as a channel for the incoming electrons in which the electrons scatter dynamically. An intuitive way of understanding this is to consider each atom as a thin lens so that as the electron wave passes through the atoms, it is focused at periodic distances [14].

It has been proven in [15] that when the electron beam leaves an isolated atom column, the image wave can be expressed to a good approximation as

$\psi(\mathbf{r}, z)=\psi(\mathbf{r}, 0)+c_{s} \phi_{s}(\mathbf{r})\left[\exp \left(-i \pi \frac{E_{s}}{E_{0}} \frac{1}{\lambda} z\right)-1\right]$,

where $\psi(\mathbf{r}, 0)$ is the incident wave, $\mathbf{r}$ describes the twodimensional vector in the plane of the image wave which is perpendicular to the beam direction, and $z$ is the column thickness (relates to the number of atoms in a column given the distance between atoms in the column is known). The incident beam energy is given by $E_{0}$ and $\lambda$ is the wavelength. The function $\phi_{s}(\mathbf{r})$ is the lowest energy bound state, the s-state, with $E_{s}$ its energy. The s-state function can be approximated by a quadratically normalized and parameterized Gaussian function [15]

$\phi_{s}(\mathbf{r})=\frac{1}{a \sqrt{2 \pi}} \exp \left(-\frac{r^{2}}{4 a^{2}}\right)$

with $a$ the column dependent width and $r=|\mathbf{r}|$. The column width is related to the energy of the s-state and is larger for a light atom column type and smaller for a heavy atom column type within the range of $0.1 \AA$ to $0.5 \AA$. The excitation coefficient $c_{s}$ is given by

$c_{s}=\int \phi_{s}^{\star}(\mathbf{r}) \psi(\mathbf{r}, 0) d \mathbf{r}$,

where the symbol $\star$ denotes the complex conjugate. For an incident plane wave, i.e. $\psi(\mathbf{r}, 0)=1, c_{s}$ equals $2 \sqrt{2 \pi} a$ when using Eq. (2). Eq. (1) is also referred to as the exit wave of an atom column to distinguish between the wave at the exit surface of the object and the wave at the image plane. Later, in Section 5, the reconstructed wave will be referred to as the experimental exit wave. A detail description will be given then.

\section{Determination of the attainable resolution}

As presented in [16], a complex plane, the so-called Argand plot, can be used to derive the structure parameters, such as the number of atoms in a column and the column height, using the pixel value at the atom column position of the image wave (or exit wave). Similarly, instead of plotting only the pixel at the column position, we can plot all the pixel values of an atom column wave in the Argand plot. According to the channelling theory, all pixels of a single atom column wave are located on a straight line in the Argand plot since they have the same phase (refer to Eq. (1) minus the incident beam wave $\phi(\mathbf{r}, 0)$ ).

An example is simulated for an isolated $\mathrm{Au}$ atom column with $z=16 \AA, a=0.13 \AA$ and $E_{s}=-210.8 \mathrm{eV}$ [17]. The incident beam energy is $300 \mathrm{keV}$. Here we assume that the microscope is free of lens aberrations. Therefore, the resolution derived is solely contributed from the atom column. The amplitude (or magnitude) of the image wave is shown in Fig. 1(a). All pixels of the image wave lie on a solid straight line in the Argand plot as shown in Fig. 1(b). If the incident beam wave is removed, meaning that the plot passes through the origin $(0,0)$, this shows that the phase of every point in the exit wave is constant as stated above. When the image wave is defocused, meaning that the image plane is at a distance $\varepsilon$ to the focal plane, the pixel values of the atom column will form a curve as shown by means of dotted lines in Fig. 1(b). The effect of defocus on the image wave can mathematically be described as a convolution product of Eq. (1) and a defocus propagator (see Appendix). Pixels closer to the column position give larger position changes in the Argand plot. This difference of position changes can also be explained from the fact that pixels closer to the column position contain more higher spatial frequency information. Thus, Fig. 1(b) also shows different defocus phase changes on the spatial frequencies contributing to the column image wave. It is observed in Fig. 1(b) that there are two stationary points (dotted circles), meaning that they do not change with defocus. One is at $(1,0)$ which is the constant background from the incident beam wave and the other one is at where the total curvature of the column wave equals zero. Note that here the surface of the column wave is defined in the three-dimensional space (bell-shaped function). The latter point will be used to define the attainable resolution. Note that this point is not strictly stationary but is a point with very little variance. This will be explained in detail in Section 4.1. In what follows, we will derive the resolution mathematically.

Substituting Eqs. (3) and (2) into Eq. (1), we can derive the curvature of the column wave using the Laplacian operator $\Delta$

$$
\begin{aligned}
\Delta \psi & =\frac{\partial^{2} \psi}{\partial x^{2}}+\frac{\partial^{2} \psi}{\partial y^{2}} \\
& =c_{s} \frac{1}{a \sqrt{2 \pi}} \exp \left(-\frac{r^{2}}{4 a^{2}}\right)\left[\exp \left(-i \pi \frac{E_{s}}{E_{0}} \frac{1}{\lambda} z\right)-1\right]\left(\frac{r^{2}}{4 a^{4}}-\frac{1}{a^{2}}\right) .
\end{aligned}
$$

Eq. (4) equals zero either when $r$ approaches a large value or when $r=2 a$. The former case corresponds to the stationary point representing the background while the latter case corresponds to the other stationary point. Thus, at

$r=r_{\Delta=0}=2 a$,

the total curvature of the image wave of an atom column equals zero within a certain defocus range (see Section 4.1).

A common definition of resolution was given by Lord Rayleigh in 1879 [1]. Rayleigh stated that the resolution is the minimum resolvable distance in the sense that two point sources are just resolvable when the central maximum of one source coincides with the first zero of the other one. The Rayleigh resolution is thus given by the distance from the central maximum to the first zero of a point spread function. The Rayleigh resolution criterion can be generalized as the distance for which the ratio of the value at the central dip of composite point spread functions to the value at the central maximum of the point spread function is equal to 0.8 . This corresponds to the original Rayleigh resolution for a rectangular aperture. Following this approach, the Rayleigh resolution $\rho_{p}$ can be derived mathematically from Eq. (1)

$0.8=2 \exp \left(-\frac{\rho_{p}^{2}}{16 a^{2}}\right)$,

from which it follows that:

$\rho_{p} \approx 4 a=2 r_{\Delta=0}$.

As a result, the resolution is directly related to the width of the atom column $a$ in the image wave. However, the width $a$ in an experimentally reconstructed wave is strongly influenced by lens aberrations such as defocus, spherical aberration, astigmatism and so on. Thus, it seems more appropriate to determine the resolution 
a

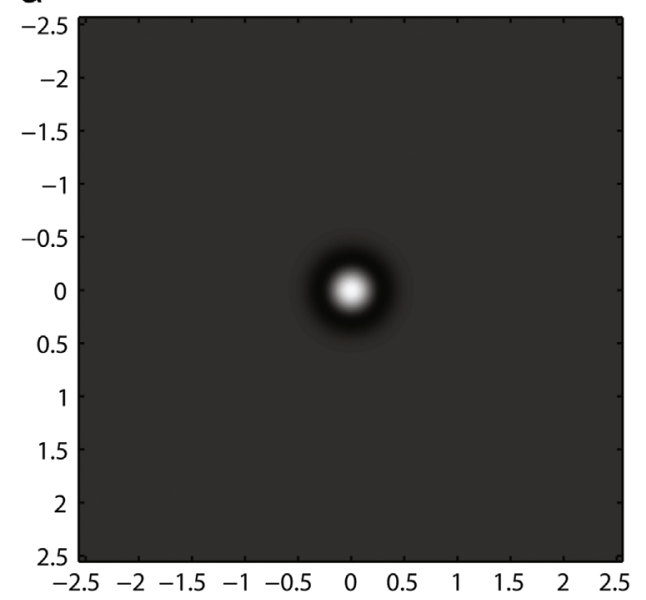

d

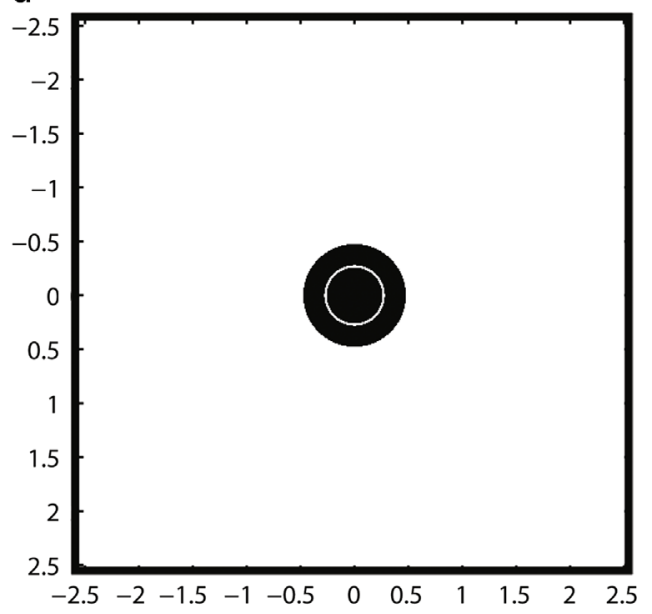

b

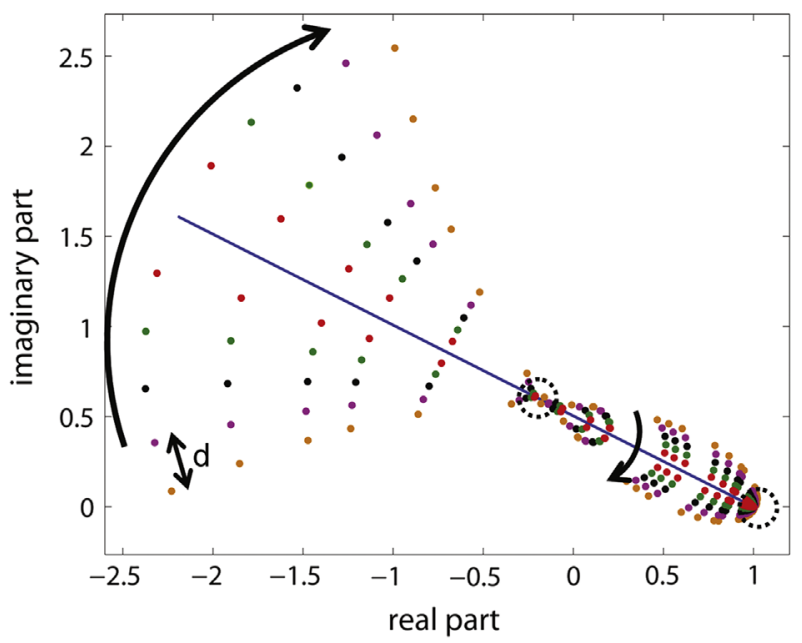

C

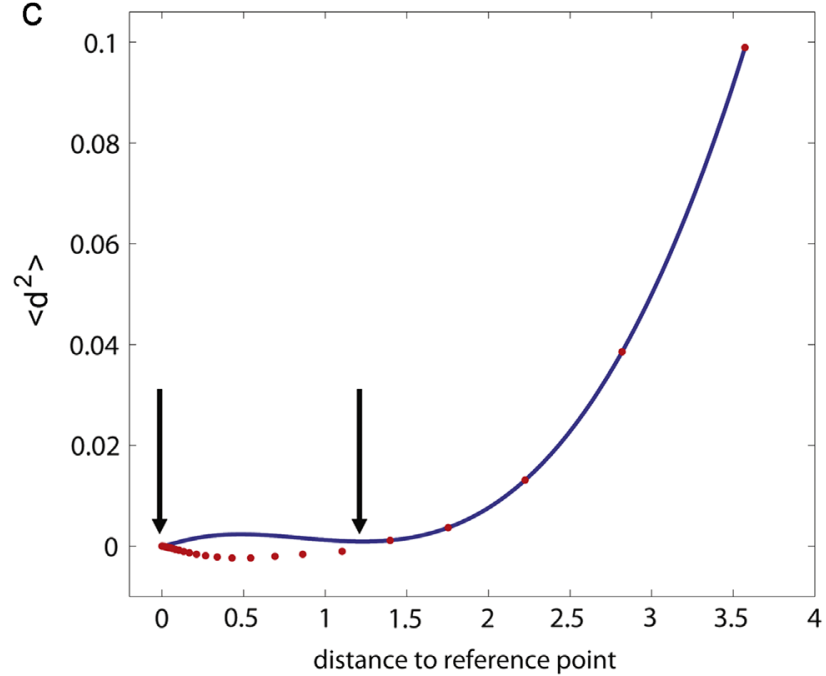

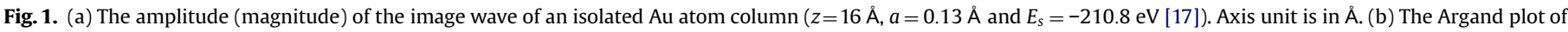

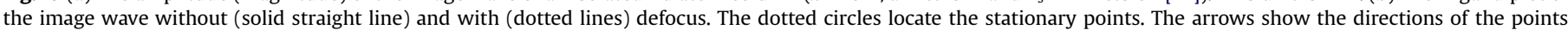

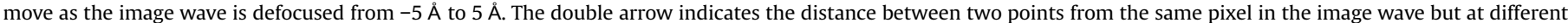

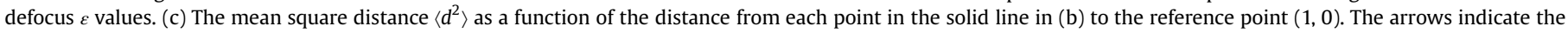

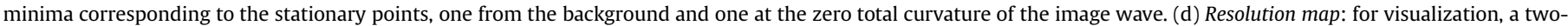
dimensional plot, size same as the image wave, presents the pixels at the stationary points in white and the rest in black. Axis unit is in Å.

from the stationary points in the Argand plot or from the reconstructed image wave itself. This will be explained and illustrated in the following subsections.

\subsection{From the Argand plot}

As described earlier, the stationary point is where the total curvature of the atom column function equals zero. In order to find this point, we can proceed as follows. First, a linear fit is applied to the points from the zero defocus image wave. The intersect with a line perpendicular to the $x$-axis is determined. This intersect point will be called the reference point. In the example shown in Fig. 1(b), we find that the fitted line intersects with $x=1$ at $(1,0)$. Second, the Argand plot is transferred to another plot where the $y$-axis represents the mean square distance $\left\langle d^{2}\right\rangle$ averaged between all defocused points from the same pixel in the image wave. The distance $d$ between two defocused points is shown as double arrows in Fig. 1(b). The $x$-axis represents the distance from each point on the solid line (from the zero-defocus exit wave) to the reference point. The plot is presented as the solid line in Fig. 1(c). The minima give the positions of the stationary points, meaning that these pixels in the image wave change minimal as the wave is defocused. Fig. 1 (c) can be improved by considering the directions in which the points in the Argand plot move as the image wave is defocused. The directions in which the points are moving are presented as curved arrows in Fig. 1(b). The points at the lower-right corner move along the opposite direction to those at the upper-left side. Thus, we present the mean square distances from those points with a negative sign and the result is shown as the dotted line in Fig. 1 (c). The stationary points can then be easily determined by the intersections of the curve with $y=0$. Later, for visualization, we can plot the pixels at the minima as white and others as black to directly locate the zero total curvature in the atom column as shown in Fig. 1 (d). The white ring clearly shows where the stationary point is in the atom column wave function. In other words, the white ring shows the pixels in the image wave that have minimal changes as the image wave is defocused. The radius of the white ring, which is $r_{\Delta=0}$, can easily be calculated as twice the column width and therefore the resolution $\rho_{p}$ equals $4 a$, being $0.52 \AA$. This type of black-and-white figure will be called a resolution map.

In Fig. 1(c), the displacement values between the two minima correspond to the outer black ring area of the atom column as 


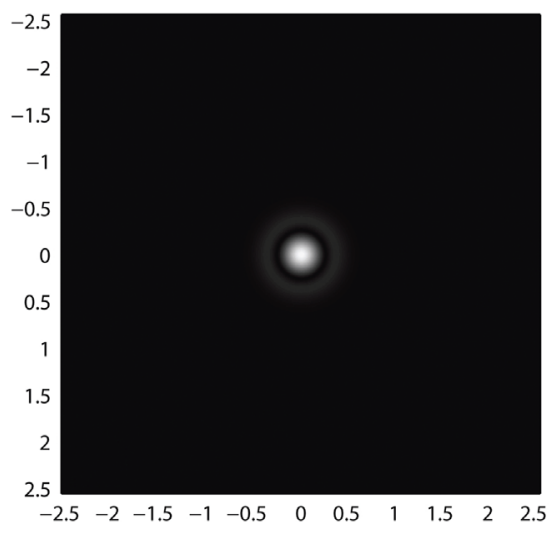

b

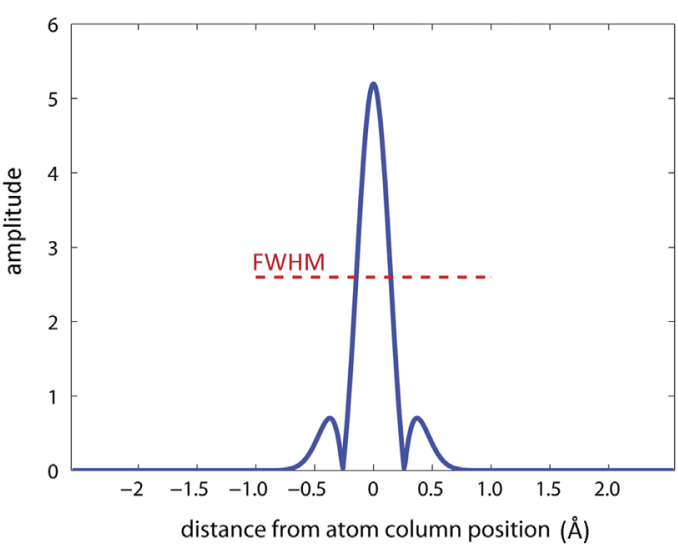

C

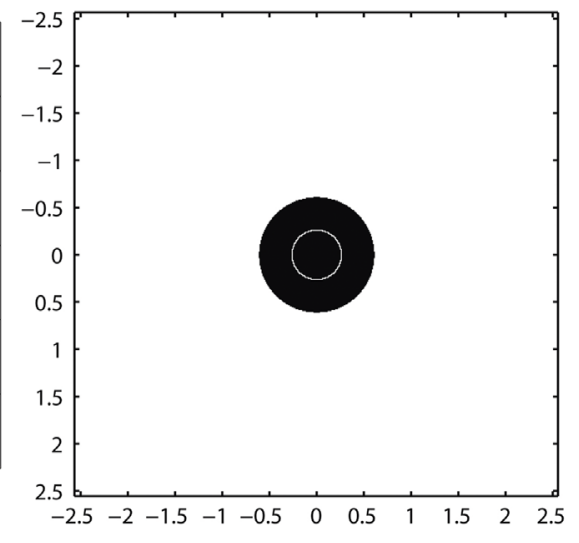

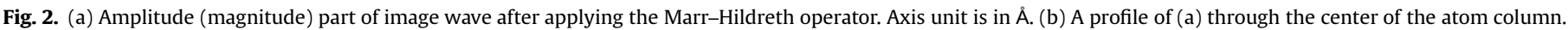
The horizontal dashed line indicates the total curvature at FWHM. (c) The resolution map in which the zero total curvature is shown in white. Axis unit is in $\AA$.

shown in Fig. 1(d). In practice, this area is strongly influenced by neighboring column waves and is sensitive to noise. The minima will then be difficult to estimate. Therefore, in the next subsection, we will show how to determine the stationary points directly from the image wave.

\subsection{From the image wave}

According to Eq. (4), the stationary point is where the Laplacian of the wave function equals zero. However, applying a Laplacian operator to the wave function may increase the fluctuation of curvature changes due to noise. Therefore, a smoothing filter, such as a Gaussian filter, is applied. This Laplacian-of-Gaussian operator is also known as the Marr-Hildreth operator [18] and is commonly used in image processing for edge detection. This operator detects the intensity change of an image $I$ through

$M=\Delta G * I$,

where $G$ is a two-dimensional Gaussian function and $*$ denotes the convolution product. $G$ acts as a smoothing filter that averages the image intensity change locally. The Marr-Hildreth operator can be described in Fourier space as

$\mathfrak{I}[\Delta G]=g^{2} \exp \left(-s g^{2}\right)$

where $\mathbf{g}$ is the two-dimensional vector in Fourier space and $g=|\mathbf{g}|$. The width of $G$ is related to $s$. Substituting Eq. (1) for $I$ and the Fourier transform of Eq. (9) into Eq. (8), we can derive that the zeros occur at $r \rightarrow \infty$ and at

$r_{\Delta=0}=\sqrt{4 a^{2}+\frac{s}{\pi^{2}}}$.

Using this relationship, the width of the atom column can be obtained and thus, the resolution $\rho_{p}$ can be derived from Eq. (6). Applying the Marr-Hildreth operator as is in fact very similar to the work of [19] where they showed that a small defocus (absence of spherical aberration) has the same effect as Eq. (9) contributing to an image, so-called Fourier imaging. This effect has been demonstrated in $[20,21]$.

In Fig. 2 we will demonstrate how the Marr-Hildreth operator is applied to an image wave to determine the resolution. Fig. 2 (a) shows the amplitude of the image wave after applying the Marr-Hildreth operator $(s=0.01)$. A profile through the center of the atom column is shown in Fig. 2(b). Similar to Fig. 1(c), the amplitude is zero at a certain distance from the atom column position and at the background. We can therefore obtain the resolution map where the zeros of the amplitude are shown in white and other values in black as presented in Fig. 2(c). This is similarly shown in Fig. 8 of [22] where the zeros of the secondorder derivative gives the inflection point of the image peak. The white ring clearly indicates where the total curvature is zero in the atom column. The radius of the ring can be estimated as $0.262 \AA$. From Eq. (10), the width of the column function $a$ can be derived as $0.13 \AA$. The resolution $\rho_{p}$ can then be estimated from Eq. (7) as $0.52 \AA ̊$.

The resolution measurement derived so far is based on the assumption that the column wave function is a Gaussian function. In case the column wave function deviates from the Gaussian shape, for instance, an exponential function, and/or in case the noise is about $10 \%$ of the maximum amplitude of $M$, the zero total curvature will not be easily detected. Therefore, we can use the full-width-half-maximum (FWHM) measurement because this part of the peak clearly surpasses the noise level. Here we do not consider the case of an even higher noise level since one may not even be able to separate individual atom columns accurately. It can be derived from Eq. (8) that the FWHM occurs at about $r=1.1 a=r_{F}$, which gives a resolution $\rho_{p}$ about $3.64 r_{F}$. We will show in the next sections that the FWHM can be determined more easily to provide the information about the resolution.

We would also like to investigate the influence of noise on the resolution determination. In order to study the noise effect on the radius of the curvature at FWHM, image waves with different amount of noise have been simulated. This is done by adding normally distributed random noise with zero mean and increasing standard deviation [23] to both real and imaginary parts of the image wave. The results indicate (not shown here) that if the standard deviation of the noise exceeds 0.15 , the radius of the curvature at FWHM cannot be easily detected which may lead to errors in the analysis. As a result, if a significant amount of noise is present, techniques to remove the noise, such as background subtraction, should be applied in advance before further analysis.

Applying the Marr-Hildreth operator on the image wave to determine the resolution seems to be a more objective method than obtaining the resolution from the Argand plot since one can determine the resolution with an arbitrary $s$. Note that $s$ should not be a large number as it compromises between noise and influence on resolution.

\section{Effects of aberrations on the resolution}

In the previous section, the resolution was determined based on perfect lenses, without lens aberrations. However, even in the 
case of electron microscopes equipped with aberration correctors, lens aberrations are not fully eliminated. Moreover, even with established programs that perform exit wave reconstruction routinely and are supposed to eliminate influence of the microscope on the exit wave, the lens aberrations are not corrected with sub-Å precision. Therefore, some lens aberrations remain in the reconstructed image wave. In this section, we will consider the common and low order lens aberrations such as defocus $\varepsilon$, spherical aberration Cs, two-fold astigmatism $A 1$ and three-fold astigmatism $A 2$ one by one and show how they affect the resolution map. Throughout this section, we obtain the resolution by applying the Marr-Hildreth operator on the image waves.

\subsection{Defocus $\varepsilon$}

The object may not be flat at the exit surface due to uneven number of atoms or located at different heights for adjacent atom columns. Thus, the column wave may not be focused at the same plane for every atom column or the exit wave cannot be reconstructed to the exact exit position of every atom column. This means that there may be a distance from the focal plane of the atom column to the image wave plane or from the exact exit position of an atom column to the exit wave plane; in other words, the column wave is defocused. This effect has been studied in [16] and a method is proposed to determine the defocus $\varepsilon$ atom column by atom column from the exit wave. Here we study the defocus effect on the resolution map.

When the image wave is defocused $\varepsilon=5 \AA$, as shown in Fig. 1 (b), the location of the zero total curvature does not change significantly. However, due to the defocus, the amplitude of $M$ is not zero within the atom column but rather a local minimum as shown in the solid line profile in Fig. 3(a). One can derive the position of this local minimum by applying Eq. (8) on a defocused image wave (see Appendix A) which gives

$r=\sqrt{4 a^{2}+\left(\frac{\varepsilon \lambda}{2 \pi a}\right)^{2}}+\left(\frac{s}{\pi^{2}}\right)$

This value does not equal $2 a$ but at small defocus the shift is insignificant. In this example, with defocus $\varepsilon=5 \AA$, the shift is about $0.03 \AA$. In case the pixel size is larger than this value, one will not be able to see such a change in resolution. Note that the pixel size is typically about $0.2 \AA$ in an experimental image. A short discussion about the pixel size is given at the end of this section. We can still obtain the resolution map as shown in
Fig. 3(b) by setting a threshold $\eta$ such that values lower than $\eta$ are shown in white otherwise in black. Note that choosing the value of $\eta$ is somewhat subjective and will affect the size of the white ring; thus, it should be chosen as close to the local minimum as possible. In Fig. 3(b), $\eta$ is set to 0.72 , from which we can estimate $r=0.29 \AA$ as expected from Eq. (11) and thus the resolution is $0.52 \AA$.

If the image wave is defocused a larger value, such as $10 \AA$, the atom column wave is blurred such that after applying the MarrHildreth operator, there is no local minimum curvature change within the column wave. This can be seen from the dashed profile shown in Fig. 3(a). Instead of a dip, there is a plateau. Therefore, even by setting a threshold $\eta=0.95$ (value of the plateau), we do not see a white ring in the resolution map similar to Fig. 3(b). As a result, it is more convenient to use the curvature at FWHM to obtain the resolution map as shown in Fig. 3(c). From this figure, we can still derive that the resolution equals $0.52 \AA$.

\subsection{Spherical aberration Cs}

A dominant aberration for electromagnetic lenses in HRTEM is the spherical aberration Cs, which causes the electron beam to be refracted stronger at the outer region of the lens than at the inner region. Though nowadays most advanced electron microscopes are equipped with a spherical aberration corrector, the standard deviation of the spherical aberration is still in the order of $1 \mu \mathrm{m}$ $[24,25]$. Therefore, we need to take this effect into account. We include two different values of $C s, 1 \mu \mathrm{m}$ and $3 \mu \mathrm{m}$, in the image waves with and without a defocus of $5 \AA$. The resolution maps are shown in Fig. 4. Following from this figure, in case there is no defocus (Fig. 4(a) and (c)), we can still set a threshold $\eta$ to determine the zero total curvature ring. In case there is defocus and spherical aberration, $\eta$ cannot be easily set. Therefore, in such case, we suggest to use the FWHM measurement as shown in Fig. 4 (b) and (d). Compared to the rings in Fig. 3, the radii of the rings in Fig. 4(a) and (c) are larger indicating that the amount of blurring is more from the spherical aberration than from defocus. Moreover, the radius in Fig. 4(c) is larger than that in Fig. 4(a) since the amount of blurring is larger. The resolutions derived from the maps in Fig. 4 are about $0.657 \AA ̊, 0.767 \AA ⿻, 0.855 \AA$, and $1.11 \AA$, respectively.

\subsection{Two-fold astigmatism A1 and three-fold astigmatism A2}

In this subsection, we consider two parasitic aberrations, twofold astigmatism $A 1$ and three-fold astigmatism $A 2$, which are

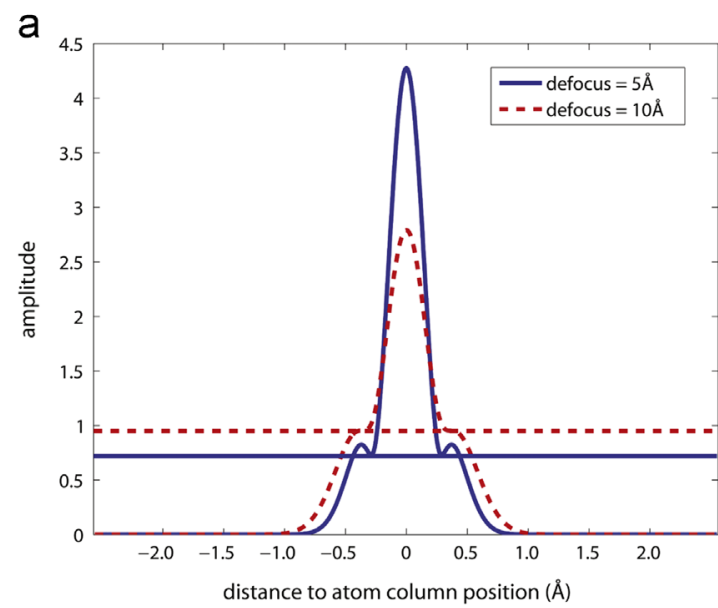

b

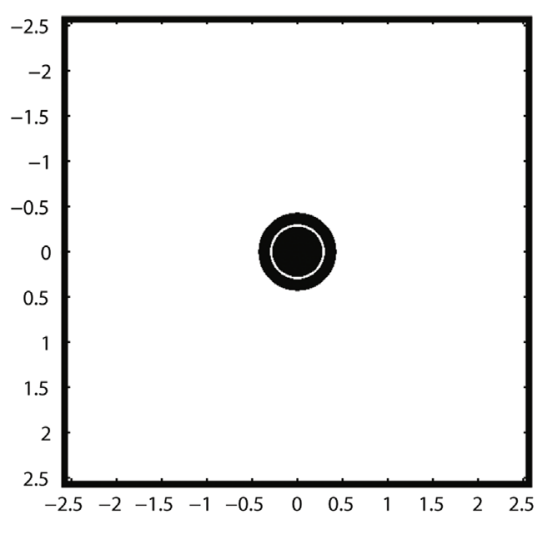

C

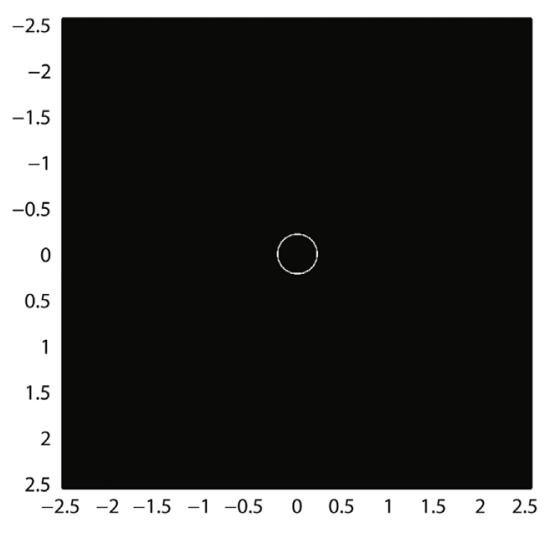

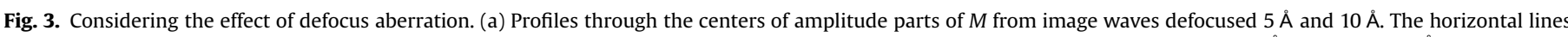

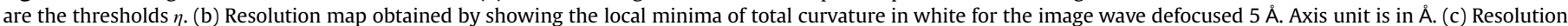
map obtained by showing the FWHM of total curvature in white for the image wave defocused $10 \AA$. Axis unit is in $\AA$. 
a

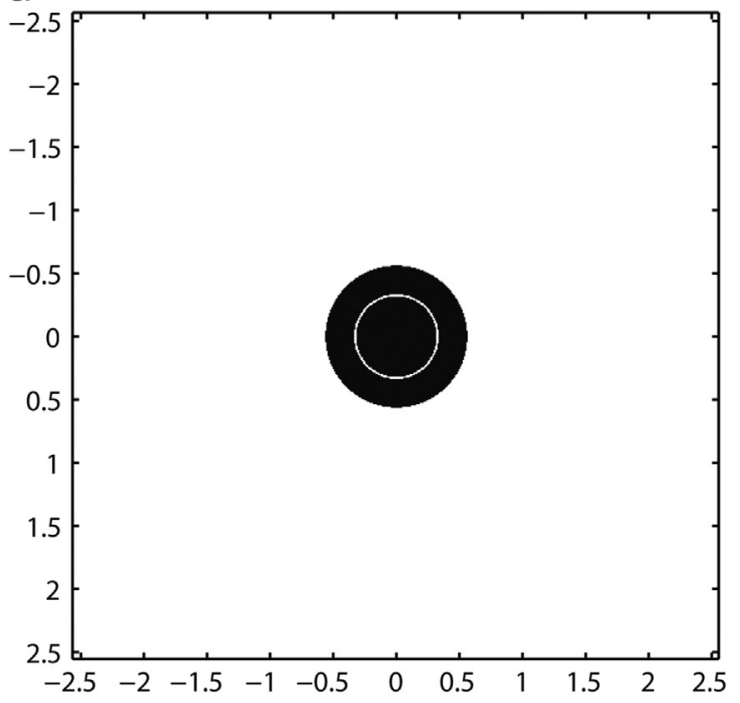

b

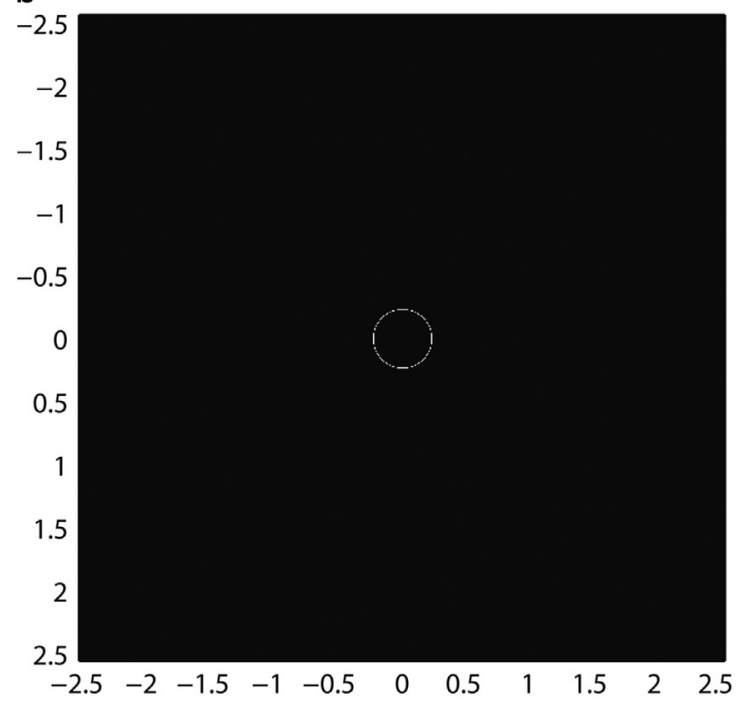

C

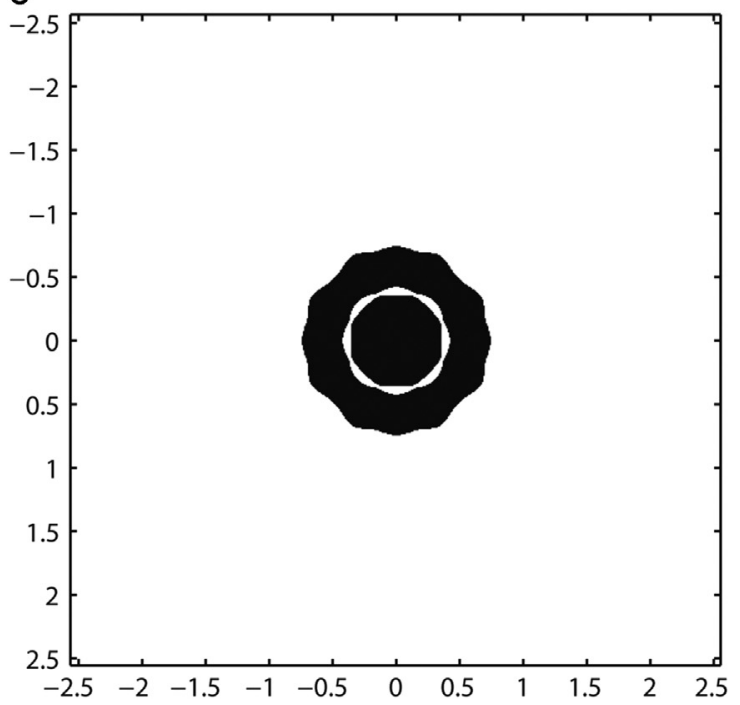

d

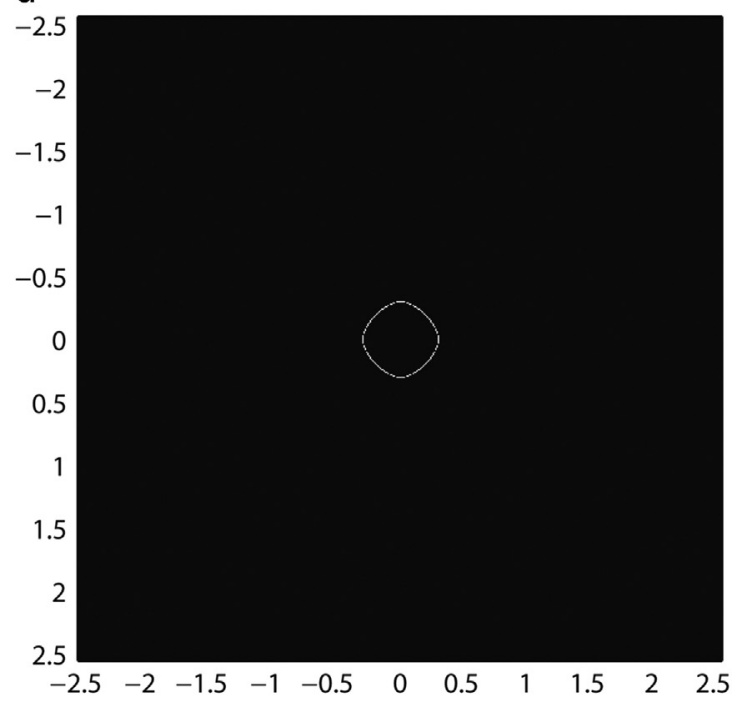

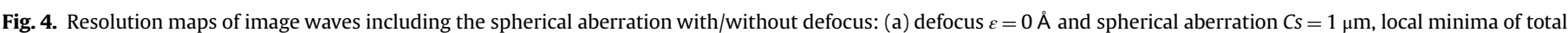

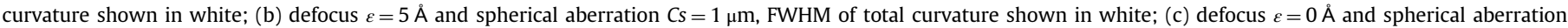

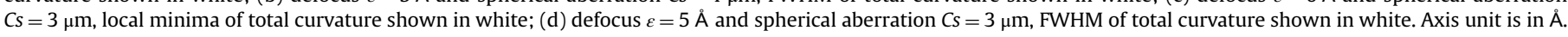

caused by deviations of the electromagnetic field from rotational symmetry. In case of two-fold astigmatism, the focal strength is different in two orthogonal directions. As a result, the column wave will be elliptically elongated. Similarly, in case of three-fold astigmatism, the column wave will form a three-fold symmetry. In order to study these aberrations separately, two defocused image waves were simulated including $A 1=10 \AA$ and $A 2=200 \AA$, respectively. Here we perform the same Marr-Hildreth operator as in previous sections on the image waves and the amplitude parts of $M$ are given in Fig. 5. It is clearly shown that the smaller pixel values (darker) form the shape of an ellipse and a triangle around the atom column, respectively. This gives the user a clear clue that astigmatism is still present. We suggest that the asymmetric aberrations should be eliminated before applying our approach to determine the resolution.

In Section 4, we studied the effects of residual aberrations on resolution maps through simulations. Defocus itself does not change the resolution significantly but in case of spherical aberration, the resolution is lower. Moreover, in the presence of astigmatism, the shape of the atom column in the resolution map turns into asymmetric shapes. We suggest that, in the future, with a routine program installed with the microscope, the user can tune the values of lens aberrations until the ring in the resolution map is the smallest and most circularly shaped. Under such microscope condition, the image wave has then the highest resolution and is most suitable for further structural analysis.

It is also shown in this section that the resolution decreases with increasing lens aberrations. Thus, the resolution of the image includes a contribution arising from the object, atoms, and the contribution from the microscope. If we can assume the atom wave as a Gaussian function (e.g. Eqs. (1) and (2)) and also the "blurring" from the microscope as a Gaussian function, then the resolution of the image $\rho_{p}$ can be approximated as [26]

$\rho_{p}^{2}=\rho_{a}^{2}+\rho_{M}^{2}$,

where $\rho_{a}$ is the resolution of the atom wave which is related to the width of the atom column $a$ and $\rho_{M}$ is the resolution of the microscope. From this equation, it follows that if one knows the width of the atom column, then one can determine the resolution of the microscope directly from the image regardless of the object under study. 
a

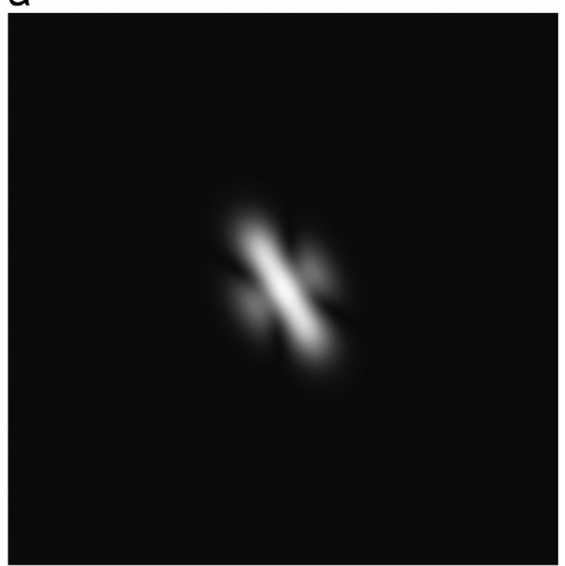

b

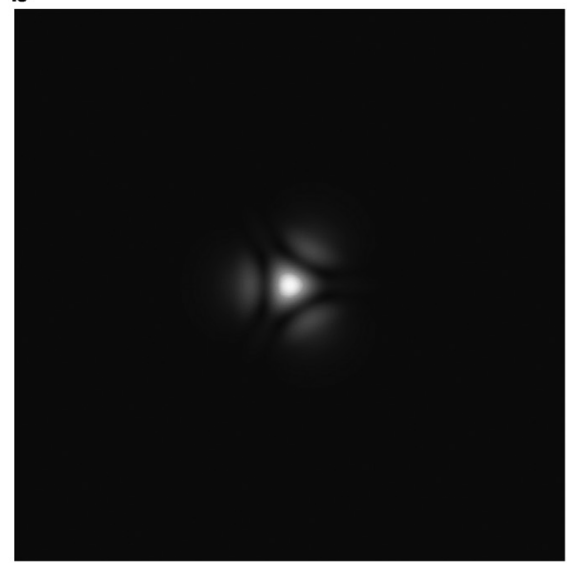

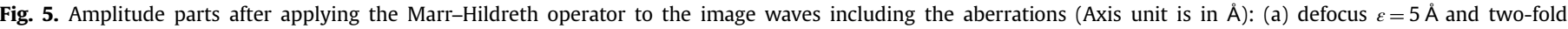
astigmatism $A 1=10 \AA\left(\phi_{22}=\pi / 3\right)$ and (b) defocus $\varepsilon=5 \AA$ and three-fold astigmatism $A 2=200 \AA\left(\phi_{33}=\pi / 3\right)$.

a

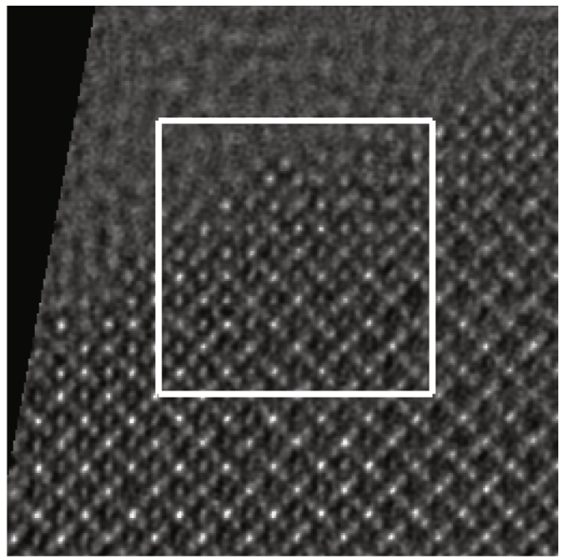

b

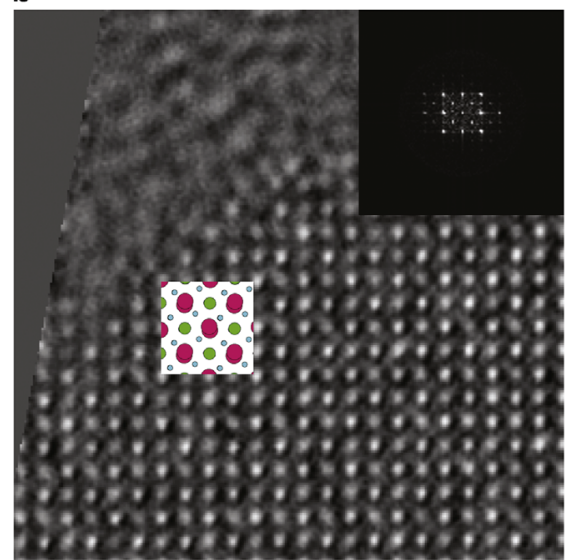

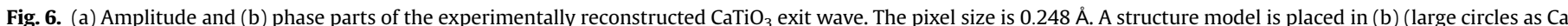

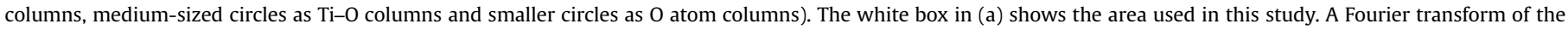
exit wave is inserted in the upper-right corner of (b).

Note that the pixel sizes used in these simulations are all $0.01 \AA$ which is an order of magnitude smaller than a typical atom column width. If the pixel size is too large (about the same order of an atom column width is considered large), the change of curvature of the atom column cannot be easily detected such that the resolution cannot be determined.

\section{A practical example}

In this section, we apply our method on an experimental $\mathrm{CaTiO}_{3}$ exit wave. An experimental exit wave is reconstructed from a series of images taken from the microscope. Due to lens apertures and spatial limitation in recording, not all scattered beams are recorded. Moreover, based on modern techniques lens aberrations cannot be estimated with sub-Å precision. Therefore, as compared to the theoretical exit wave described in Section 2, a reconstructed exit wave includes limited structure information about the object and may still contain "residual" lens aberrations. In this experiment, an aberration-corrected transmission electron microscope, FEI Titan ${ }^{3} 50-80$, operating at $300 \mathrm{keV}$ was used to record a series of high resolution images with defocus ranging from $270 \AA$ to $-186 \AA$ with a defocus step of $24 \AA$. For details on the microscope parameters and the sample under study, we refer to [27]. The reconstruction of the exit wave was carried out using the TrueImage software $[28,29]$. The amount of lens aberrations is minimized using standard techniques in TrueImage. The amplitude and phase of the exit wave are shown in Fig. 6(a) and (b), respectively, which is reconstructed up to a resolution of $0.8 \AA$. This value is in general calculated from the microscope transfer function with the given lens parameters. A structure model is shown in Fig. 6(b). A Fourier transform of the exit wave is inserted in Fig. 6(b). The white box inserted shows the area used in this study. The area under study is chosen close to the edge so that the thickness variation is minimized.

In Section 5.1, the method will be described applying on the experimental data. Then in Section 5.2, the results will be discussed.

\subsection{Data analysis}

The pixel size of this exit wave as delivered from the camera system is $0.248 \AA$, which is about the same order as the column width. Although this sampling obeys the Nyquist criterion so that no information is lost, it cannot be used for further numerical treatment. Therefore, we first re-sample the exit wave eight times smaller using a spline interpolation method. In this section, we present the determination of the resolution according to the methods described in Sections 3.1 and 3.2 using the Argand plot and the Marr-Hildreth operator, respectively. 


\subsubsection{From the Argand plot}

As described in Section 3.1, we can obtain the resolution by determining the stationary points in the Argand plot from a set of defocused exit waves. The exit wave was defocused from $-100 \AA$ to $100 \AA$ with a defocus step of $1 \AA$. Fig. 7(a) shows the Argand plot of a $\mathrm{Ca}$ atom column close to the edge from several defocused exit waves (for visualization). Similar to Fig. 1(b), the pixels closer to the column center have larger displacements between defocused points. Also, those points move along the opposite direction to those from pixels further away from the column center. We can somewhat visualize that there are dense points (shown with dotted circle in Fig. 7(a)) where the displacement is minimal. Then, we can translate this figure into Fig. 7 (b) similarly as Fig. 1(c). In this experimental example, we directly take into account the direction of displacement so that the mean square displacements from pixels further away from the column center has a negative sign. As shown, either from the lower-left corner of Fig. 7(a) or from the negative points in (b), pixels corresponding to the area of the atom column further away from the column center are strongly influenced by neighboring column waves and noise. This makes the intersection of the curve with $y=0$ in Fig. 7(b) more difficult to determine. In order to find the intersection, we fit a 3rd-order polynomial to the points which is shown as the solid curve in the figure. Then, the pixels corresponding to the points in the flat region (between the two vertical lines) are colored in white while others are in black as shown in Fig. 7(c). We can clearly see a ring around the atom column. From this figure, we can estimate the radius of the ring about $0.65 \AA$ and thus the resolution about $1.3 \AA$.

From this example, we see that since there is no clear intersection of the curve with $y=0$ in Fig. 7(b), the ring in Fig. 7(c) is thick. This is due to the fact that, as stated earlier in Section 3.1, the pixels corresponding to the flat region are strongly influenced by neighboring waves and noise. As a result, the ring and thus the resolution cannot be estimated precisely. Therefore, we will directly determine the resolution by using the Marr-Hildreth operator as follows.

\subsubsection{Marr-Hildreth operator}

The amplitude parts after applying the Marr-Hildreth operator with $s=0.03$ are shown in Fig. 8(a). Since the pixels in between atom columns are about the same values as those at the local minima of the atom columns, it is more suitable to obtain the total curvature at FWHM as shown in Fig. 8(b). The FWHM is obtained locally atom column by atom column. From the resolution map, we can see that there are white rings at the $\mathrm{Ca}$ and $\mathrm{Ti}-\mathrm{O}$ atom columns and also in between. The white contrast in between these columns result from the $\mathrm{O}$ atom columns and also, possibly, noise. As a result, these white contrast as well as columns near the border is filtered before further analysis. This can be done by creating a user-created mask for filtering. The filtered resolution map is shown in Fig. 8(c). Keeping only the white rings at the Ca and $\mathrm{Ti}-\mathrm{O}$ atom columns, the radii of the rings in the resolution map are obtained. The radius is determined by the distance of the white pixel around the column to the center maximum. The radii for both types of atom columns are presented as histograms in Fig. 8(d). From this histogram, we can derive the mean radius for Ca column is about $0.333 \AA$, and that for Ti-O is about $0.354 \AA$. Therefore, we can estimate the resolution $\rho_{p}$ as $1.24 \AA$. This value is larger than the value given from the software, $0.8 \AA$.

If the amount of noise in the experimental data is high, we can apply a simple filtering procedure called lattice averaging. This is applicable when assuming the area under study has about the same thickness and the same surface profile. This procedure is done by refining the Fourier spots as shown in Fig. 6(b). According to simulations, given the known positions of the Fourier spots, Gaussian functions are fitted to the intensities of the experimental Fourier spots. The averaged lattice is then obtained by Fourier transforming the fitted model of the Fourier spots as shown in Fig. 9(a). Then the same Marr-Hildreth operator with $s=0.03$ is applied to this averaged lattice. The amplitude part after applying the operator is shown in Fig. 9(b). From this figure, we can see that the minima around the atom columns are clearly presented, even around some $\mathrm{O}$ atom columns. We apply a mask filter to remove columns near the border and the pixels in between the $\mathrm{Ca}$ and Ti-O atom columns. Also, the $\mathrm{O}$ atom columns are removed since we are only interested in comparing the results with Fig. 8. We can again obtain the resolution map by finding the total curvature at FWHM of the Ca and Ti-O atom columns as shown in Fig. 9(c). The histograms of the radius of the rings are given in Fig. 9(d). The mean radius for Ca column is about $0.376 \AA$, and that for Ti-O is about $0.296 \AA$. Therefore, we can estimate the resolution $\rho_{p}$ as $1.22 \AA$.

\subsection{Discussions}

In the previous section, we have demonstrated how to determine the resolution in practice. We have used an experimentally reconstructed exit wave. The reconstructed exit wave is supposed a

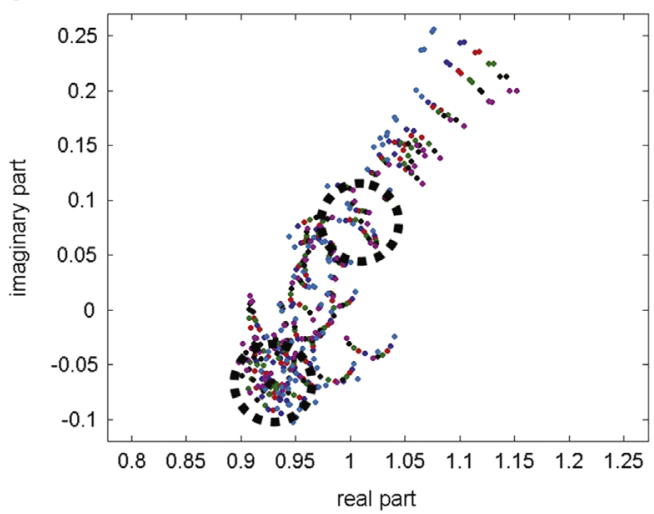

b

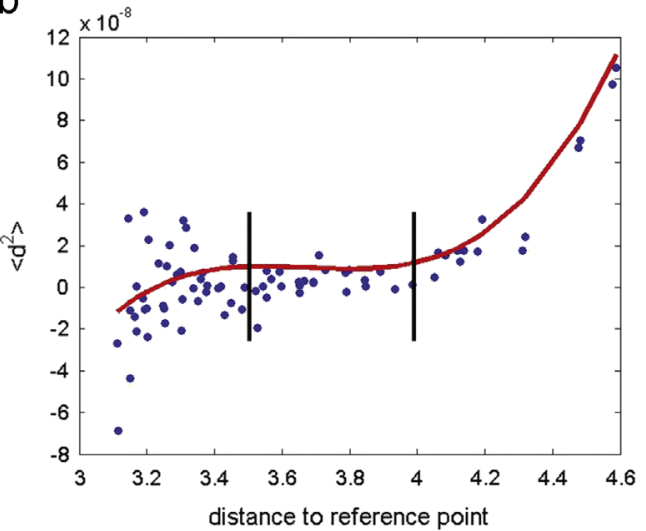

C

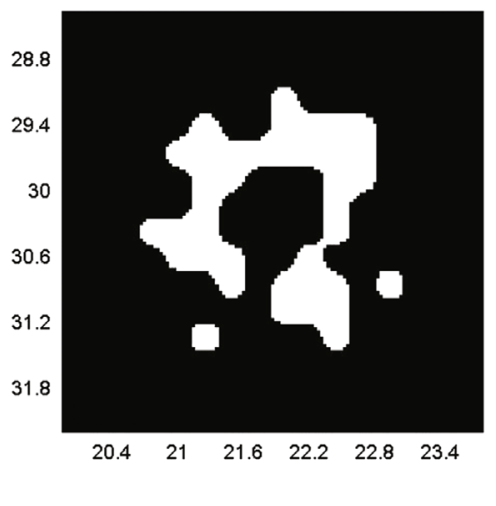

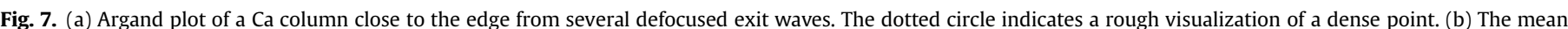

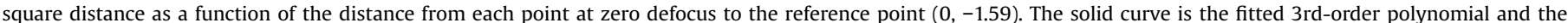

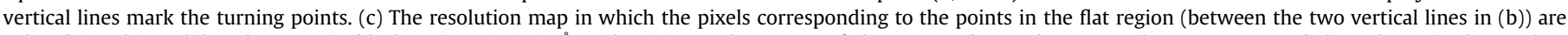

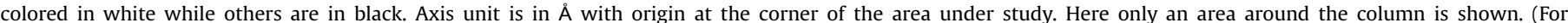
interpretation of the references to color in this figure caption, the reader is referred to the web version of this paper.) 
a

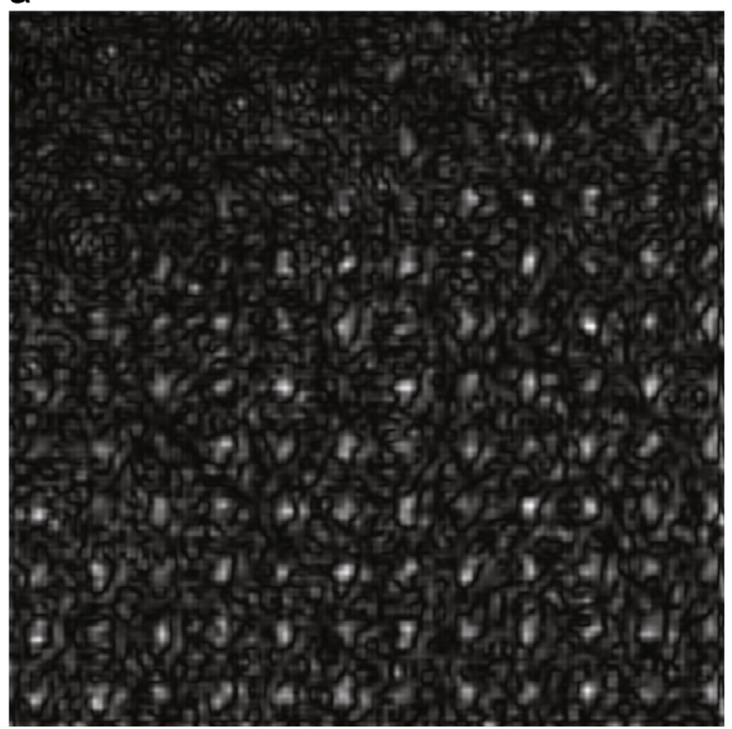

C

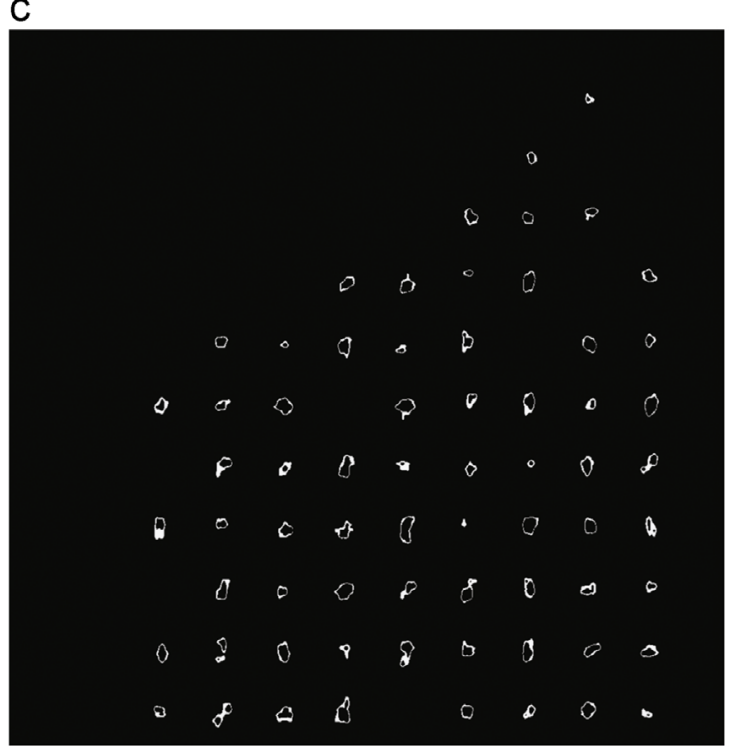

b

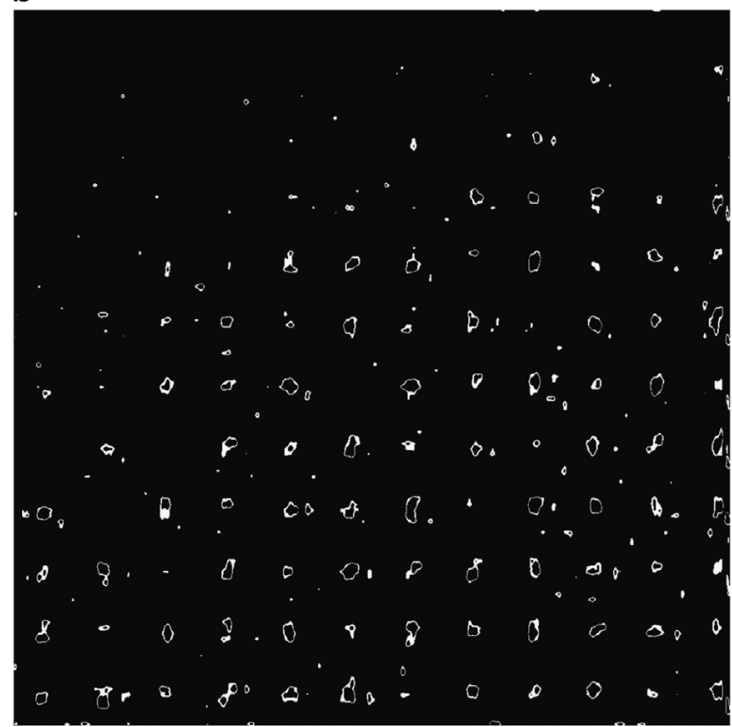

d

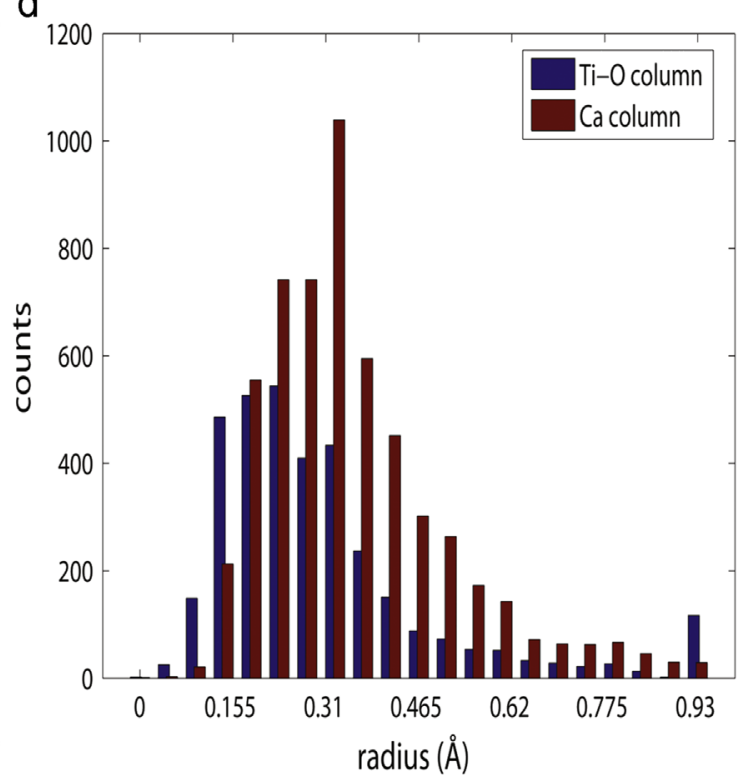

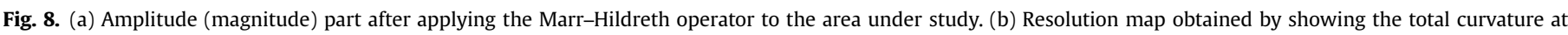

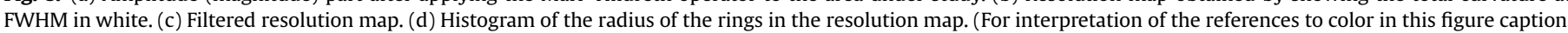
the reader is referred to the web version of this paper.)

to be free of microscope aberrations except of local defocus which may vary atom column by atom column. However, as shown in Fig. 8 (c), not all the rings around the columns are circularly shaped. Thus, we present the resolution as an averaged measurement from all the atom columns in the area under study. The resolution obtained, about $1.2 \AA$, is larger than the value given from the software, $0.8 \AA$. The difference between these two values mainly results from the atom column itself. This means that the image is a combination of the electron beam spreading from the atoms and from the microscope lenses. Therefore, the resolution of an image is a combination of the resolution of the atoms, which is limited by the width of the atoms, and the resolution of the microscope. To demonstrate this, a simulation study has been carried out. A simulated $\mathrm{CaTiO}_{3}$ reconstructed exit wave has been generated using a series of through-focal simulated images with the same microscopic conditions as described in the beginning of Section 5. The same analysis procedure has been applied on this simulated reconstructed exit wave. The resolution thus determined equals about $1 \AA$, which is larger than the expected contribution of $0.8 \AA$ if only instrumental conditions would contribute. The simulation images did not include experimental conditions such as noise or image shift, which may also explain why the resolution obtained from the simulated images is slightly better than that from the experimental images. In this simulation, we have also applied the analysis procedure on the simulated exit wave which is free of microscope aberrations. The resolution determined equals about $0.53 \AA$ which is contributed only from the atoms. These resolution measurements are in good agreement with Eq. (12). Thus, as suggested in the end of Section 4, if one can know precisely the width of the column wave which is dominated by the s-state wave, one can determine the resolution of the microscope directly.

This method may also be applied on reconstructed image waves. The main difference between a reconstructed image wave and a reconstructed exit wave is that the former is the wave at the image plane which contains the information about the microscope lenses. As stated earlier in Section 4, the user may use the shape and size of the rings in the resolution map to minimize the total 
a

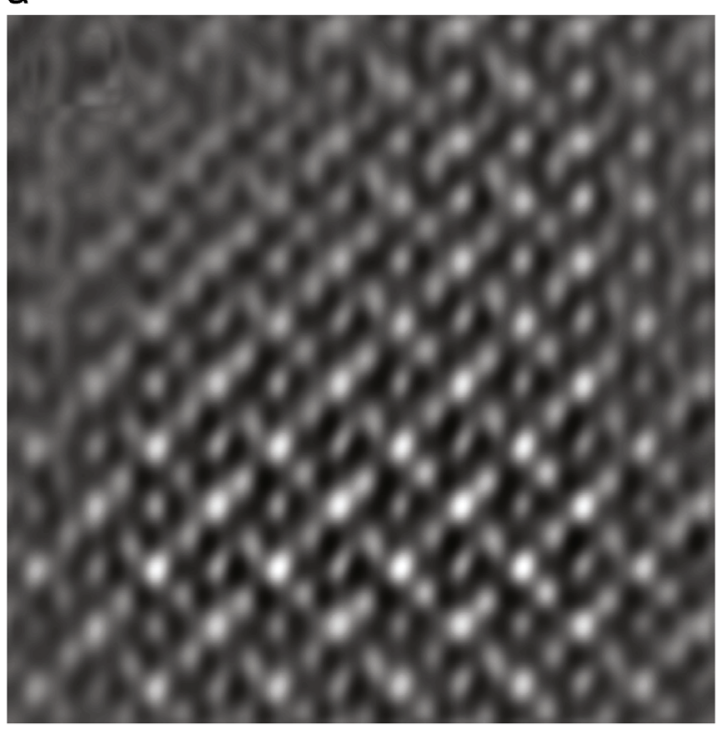

C

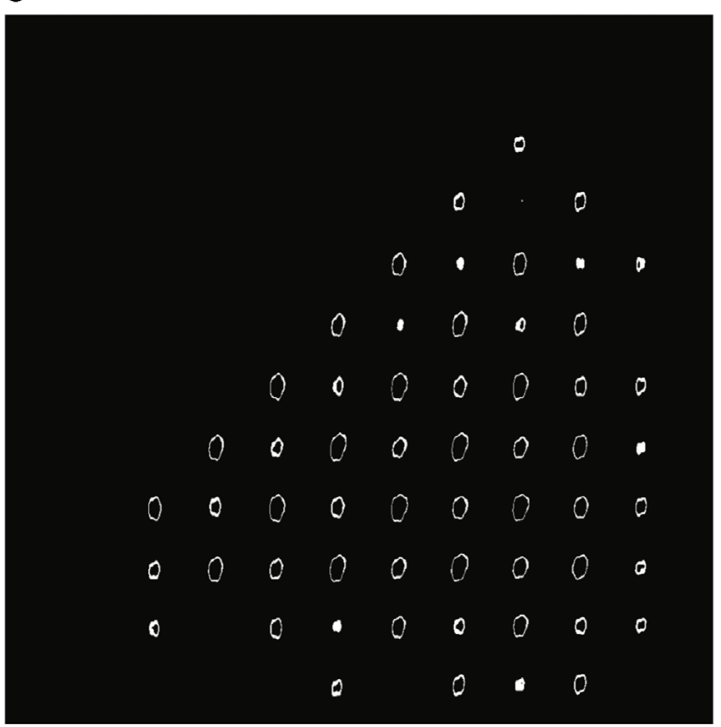

b

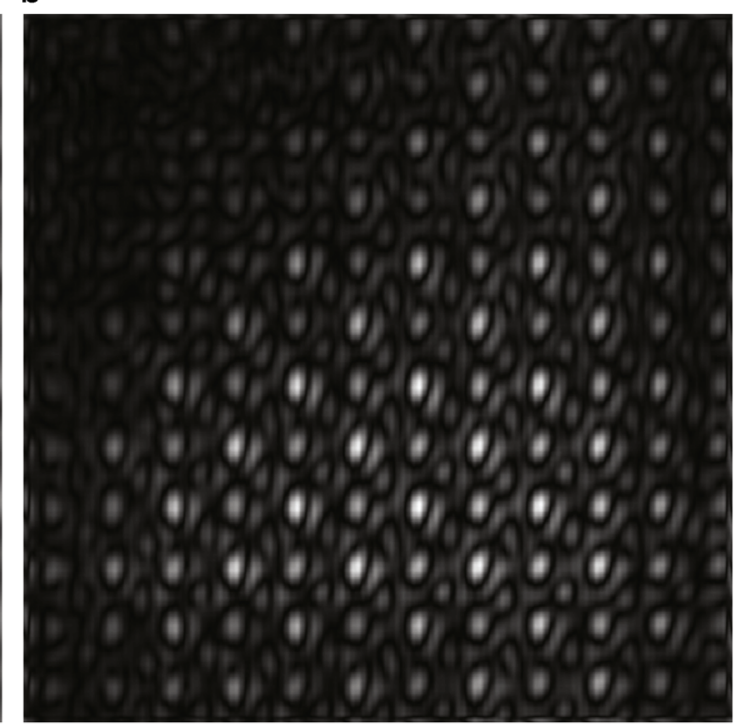

d

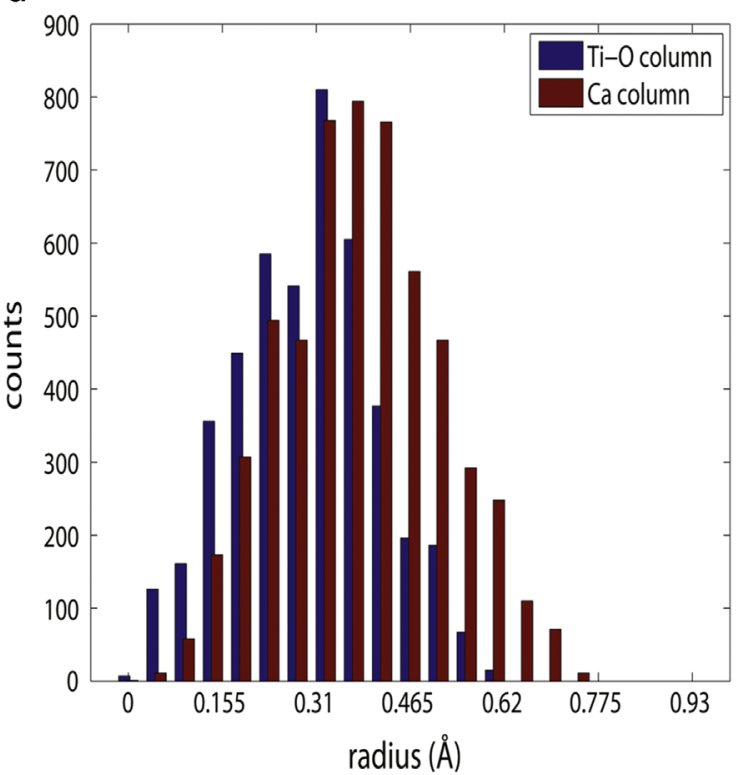

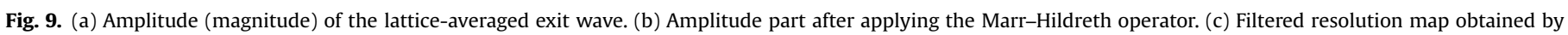

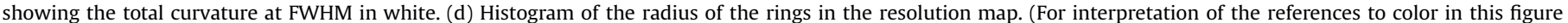
caption, the reader is referred to the web version of this paper.)

aberration effect. Theoretically, the resolution obtained from a reconstructed image wave scales with a factor resulting from the symmetric lens aberrations as compared with that obtained from a reconstructed exit wave. However, as shown in Section 4, the radius of the rings in the resolution map increases as the amount of aberration increases. It is evident that, in case of either large defocus, spherical aberration or both, the rings may overlap or become difficult to interpret due to phase wrapping. Therefore, we suggest that the method is applied on a reconstructed exit wave where most lens aberrations are eliminated. It should be noted that, however, that if the images are not aligned properly (which is unlikely using modern exit wave reconstruction program), this may contribute to an extra boardening of the reconstructed atom wave function which may result in a larger resolution value. This method is of course not suitable to apply on a recorded image itself. An image is the intensity distribution of electron counts recorded by the detector. The image formation through the detector is a complicated and nonlinear process and as such, the wave information is mixed in a nonlinear way. As a result, the resolution information is also mixed in the image.

One may suggest that the resolution can be related to the most distant spot from the center in a diffraction pattern or the Fourier transform of the exit wave. In case of an amorphous thin object, the electron channelling effect does not occur and the electrons are kinematically scattered. If this would be a perfect white noise object with a constant spectrum, the diffractogram would directly reveal the transfer function. The resolution can then be determined from the Thon rings and the visibility can be improved by Young's fringes [5,6]. However, the object is never a pure phase object because it consists of atoms so that the transfer function is dampened not only by the aberrations of the microscope but also by the scattering factor of the constituting atoms. Therefore, one uses heavy atoms such as tungsten. Even for such atoms the damping is already significant when one enters the sub-Å regime of advanced Cs-corrected electron microscopes. Also, there is influence from the microscope where mechanical vibrations may 
easily damp the intensities at high spatial frequencies [2]. One also has to avoid using nonlinear contributions in the diffractograms because they are not a true proof of resolution. Moreover, there appears to be no standard technique to determine the most distant spot in a diffractogram. In X-ray diffraction, a common criterion applied is that the intensity of a reliable spot should be at least three times larger than the standard deviation of the intensity [30]. Applying such criteria on the Fourier transform of the exit wave as shown in Fig. 6(b), it is suggested that the most distant spot corresponds with a resolution of about $1.21 \AA$. In this example, this value is about the averaged resolution we obtained in the previous subsection.

\section{Conclusions}

In this paper, we have introduced an alternative method to determine the resolution directly in an object-independent way. We have observed that the pixels in the atom column plotted in an Argand plot pass through two stationary points when the column wave is being defocused in a small defocus range. One stationary point is at the background, whereas the other one, based on the channelling theory, is at the zero total curvature of the atom column (considering the atom column surface as a bell-shape function in the three-dimensional space). Moreover, using the Rayleigh criterion, we can relate the attainable resolution to the zero total curvature. An objective method is proposed to determine the total curvature of the atom column; that is, by applying a Marr-Hildreth operator on the column wave. We have suggested, if the zero total curvature cannot be easily detected (may be because of noise), we can also use the FWHM measurement to relate to the resolution. Both criteria yield a circular ring around the atom column position which can easily be visualized in white on a black constant. We have demonstrated that with the presence of lens aberrations, either the size of the ring is enlarged or the shape is changed. We suggest that, in the future, with a routine program installed with the microscope, the size and/or the shape of the ring may be used as a fingerprint for a microscopist to minimize aberrations by adjusting the microscopic lenses to obtain the smallest circular ring possible. Therefore, the image wave has then the highest resolution and is most suitable for further structural analysis.

The method has been applied to an experimentally reconstructed exit wave of $\mathrm{CaTiO}_{3}$. We have shown that the resolution determined using the proposed method is about $1.2 \AA$ which is larger than the one given from the software. This difference proves that the resolution of an image cannot be determined solely from the electron microscope. Only when the object would be a perfect white noise object or when the resolution of the microscope is much larger that the width of the atoms, the resolution of an image can be directly determined from the diffractogram or from the exit wave. The results presented reflect the statement in the introduction that the resolution of an image is set by the resolution of the microscope and by the object itself which is much smaller using a crystalline object in a channeling condition than with the use of an amorphous film. We also suggested that the resolution of the microscope can be directly determined given the width of the atom column known. If, in the near future, the microscope reaches picometer resolution, then the resolution will ultimately be limited by the width of the focusing channelling wave.

\section{Acknowledgments}

A. Wang, S. Van Aert and D. Van Dyck are grateful to the Fund for Scientific Research-Flanders (FWO) (project no. G.0064.10,

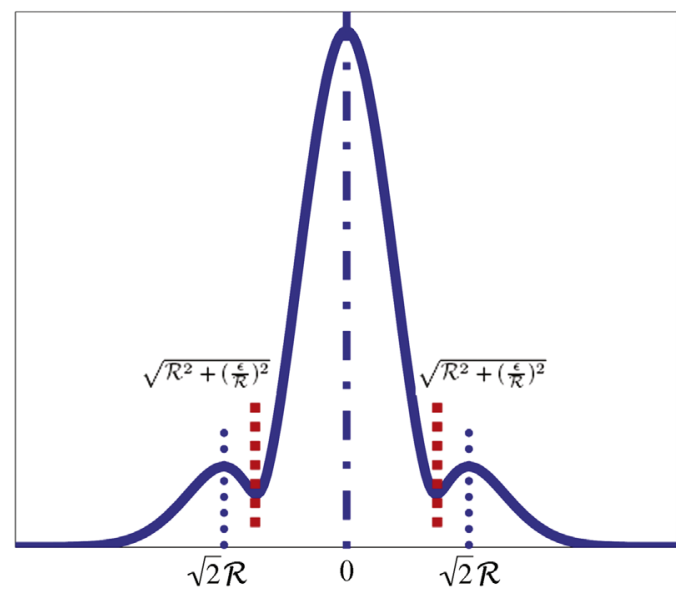

Fig. A1. Line profile of the amplitude part of a defocused image wave after applying the Marr-Hildreth operator. The positions of the local extrema are indicated: ••• local maximum, ma local minimum and at the center is the maximum.

G.0393.11 and G.0374.13). S. Turner acknowledges financial support from the Fund for Scientific Research-Flanders (FWO) for a post-doctoral fellowship. The Titan microscope used for this study was partially funded by the HERCULES foundation of the Flemish Government. The research leading to these results has received funding from the European Union Seventh Framework Programme [FP7/2007-2013] under Grant agreement no. 312483 (ESTEEM2).

\section{Appendix A}

In this appendix, we derive the radius of the ring in the resolution map as a function of defocus $\varepsilon$. The effect of defocus on the image wave for a particular atom column can mathematically be described as a convolution product of Eq. (1) and the defocus propagator $p(\mathbf{r})$

$\left.\psi_{\varepsilon}(\mathbf{r}, z)=\psi_{(} \mathbf{r}, z\right) * p(\mathbf{r})$

The defocus propagator, described by Fresnel diffraction as the wave propagates through vacuum, is given by

$p(\mathbf{r})=\mathfrak{J}^{-1}\left[\exp \left(-i \pi \varepsilon \lambda g^{2}\right)\right]$

where $\mathfrak{J}^{-1}$ denotes the inverse Fourier transformation and $g$ the length of the two-dimensional reciprocal wave vector $\mathbf{g}$. Using the convolution theorem, it can be shown that the defocused image wave of one atom column is given by

$$
\begin{aligned}
\psi_{\varepsilon}(\mathbf{r}, z)= & +c_{s} \frac{2 \sqrt{2 \pi} a}{4 \pi a^{2}+i \varepsilon \lambda} \exp \left(-\frac{(\mathbf{r}-\boldsymbol{\beta})^{2}}{4 a^{2}+i \frac{\varepsilon \lambda}{\pi}}\right) \\
& \times\left[\exp \left(-i \pi \frac{E_{s}}{E_{0}} \frac{z}{\lambda}\right)-1\right] .
\end{aligned}
$$

Applying the Laplacian operator onto Eq. (A.3), we can derive

$$
\begin{aligned}
\Delta \psi_{\varepsilon}(\mathbf{r}, z)= & 32 a^{2} \frac{1}{\mathcal{R}^{2}+i \epsilon} \exp \left(-\frac{r^{2}}{\mathcal{R}^{2}+i \epsilon}\right)\left[\frac{r^{2}}{\left(\mathcal{R}^{2}+i \epsilon\right)^{2}}-\frac{1}{\mathcal{R}^{2}+i \epsilon}\right] \\
& \times\left[\exp \left(-i \pi \frac{E_{s}}{E_{0}} \frac{z}{\lambda}\right)-1\right]
\end{aligned}
$$

with $\mathcal{R}=2 a$ and $\epsilon=\varepsilon \lambda / \pi$. The local extrema can be found at $r=0$ (maximum), $\sqrt{2} \mathcal{R}$ (local maximum) and $\sqrt{\mathcal{R}^{2}+(\epsilon / \mathcal{R})^{2}}$ (local minimum). The locations of these extrema are marked in Fig. A1. When applying the Marr-Hildreth operator (Eq. (8)), the radius at 
the local minimum scales with $s$ similarly to Eq. (10), as

$r_{\Delta=\min }=\sqrt{\mathcal{R}^{2}+\left(\frac{\epsilon}{\mathcal{R}}\right)^{2}+\left(\frac{S}{\pi^{2}}\right)}$.

\section{References}

[1] Lord Rayleigh, Investigations in optics, with special reference to the spectroscope, Philosophical Magazine VIII (1879).

[2] M.A. O'Keefe, "Resolution" in high-resolution electron microscopy, Ultramicroscopy 47 (1992) 282-297.

[3] J.C.H. Spence, Experimental High-Resolution Electron Microscopy, 2nd edition, Oxford University Press, New York, 1988.

[4] A.F. de Jong, D. Van Dyck, Ultimate resolution and information in electron microscopy II. The information limit of transmission electron microscopes, Ultramicroscopy 49 (1993) 66-80.

[5] H. Bakker, A. Bleeker, P. Mul, Design and performance of an ultra-highresolution $300 \mathrm{kV}$ microscope, Ultramicroscopy 64 (1996) 17-34.

[6] D. Van Dyck, H. Lichte, K.D. van der Mast, Sub-Ångstrom structure characterisation: the Brite-Euram route towards one Ångstrom, Ultramicroscopy 64 (1996) 1-15.

[7] $\mathrm{H}$. Rose, Outline of a spherically corrected semiaplanatic medium-voltage transmission electron microscopy, Optik 85 (1990) 19-24.

[8] P.E. Batson, N. Dellby, O.L. Krivanek, Sub-ångstrom resolution using aberration corrected electron optics, Nature 418 (2002) 617-620.

[9] M.A. O'Keefe, C.J.D. Hetherington, Y.C. Wang, E.C. Nelson, J.H. Turner C. Kisielowski, J.O. Malm, R. Mueller, J. Ringnalda, M. Pan, A. Thust, SubAngstrom high-resolution transmission electron microscopy at $300 \mathrm{keV}$, Ultramicroscopy 89 (2001) 212-241.

[10] C. Kisielowski, C.J.D. Hetherington, Y.C. Wang, R. Kilaas, M.A. O'Keefe, A. Thust, Imaging columns of the light elements carbon, nitrogen and oxygen with subÅngstrom resolution, Ultramicroscopy 89 (2001) 243-263.

[11] M. Lentzen, B. Jahnen, C.L. Jia, A. Thust, K. Tillmann, K. Urban, High-resolution imaging with an aberration-corrected transmission electron microscope, Ultramicroscopy 92 (2002) 233-242.

[12] S. Van Aert, J. Chen, D. Van Dyck, Linear versus non-linear structural information limit in high-resolution transmission electron microscopy, Ultramicroscopy 110 (2010) 1404-1410.

[13] D. Van Dyck, M. Op de Beeck, A simple intuitive theory for electron diffraction, Ultramicroscopy 64 (1996) 99-107.

[14] S. Van Aert, P. Geuens, D. Van Dyck, C. Kisielowski, J.R. Jinschek, Electron channelling based crystallography, Ultramicroscopy 107 (2007) 551-558.
[15] P. Geuens, D. Van Dyck, The S-state model: a work horse for HRTEM, Ultramicroscopy 93 (2002) 179-198.

[16] A. Wang, F.R. Chen, S. Van Aert, D. Van Dyck, Direct structure inversion from exit waves. Part I: theory and simulations, Ultramicroscopy 110 (2010) 527-534.

[17] P. Geuens, J.H. Chen, A.J. den Dekker, D. Van Dyck, An analytic expression in closed form for the electron exit wave, Acta Crystallographica, Section A 55 Supplement, Abstract P11.OE.002 (1999).

[18] D. Marr, E. Hildreth, Theory of edge detection, Proceedings of the Royal Society of London B 207 (1980) 187-217.

[19] J.M. Cowley, A.F. Moodie, Fourier images: I-the point source, Proceedings of the Physical Society. Section B 70 (1957) 486-496.

[20] D.F. Lynch, A.F. Moodie, M.A. O'Keefe, n-Beam lattice images. V. The use of the charge-density approximation in the interpretation of lattice images, Acta Crystallographica A 31 (1975) 300-307.

[21] M.A. O'Keefe, J.V. Sanders, The phase contrast component of lattice images of a zeolite crystal, Optik 46 (1976) 421-430.

[22] M.A. O'Keefe, Seeing atoms with aberration-corrected sub-Ångstrom electron microscopy, Ultramicroscopy 108 (2008) 196-209.

[23] A.H. Buist, A. van den Bos, M.A.O. Miedema, Optimal experimental design for exit wave reconstruction from focal series in TEM, Ultramicroscopy 64 (1996) 137-152.

[24] M. Lentzen, Progress in aberration-corrected high-resolution transmission electron microscopy using hardware aberration correction, Microscopy and Microanalysis 12 (2006) 191-205.

[25] A.R. Lupini, A.Y. Borisevich, J.C. Idrobo, H.M. Christen, M. Biegalski, S.J. Pennycook, Characterizing the two- and three-dimensional resolution of an improved aberration-corrected STEM, Microscopy and Microanalysis 15 (2009) 441-453.

[26] D. Van Dyck, S. Van Aert, A.J. den Dekker, A. van den Bos, Is atomic resolution transmission electron microscopy able to resolve and refine amorphous structures? Ultramicroscopy 98 (2003) 27-42.

[27] S. Van Aert, S. Turner, R. Delville, D. Schryvers, G. Van Tendeloo, E.K.H. Saljeh, Direct observation of ferrielectricity at ferroelastic domain boundaries in $\mathrm{CaTiO}_{3}$ by electron microscopy, Advanced Materials 24 (2012) 523-527.

[28] W. Coene, A. Thust, D. Van Dyck, M. Op de Beeck, Maximum-likelihood method for focus-variation image reconstruction in high resolution transmission electron microscopy, Ultramicroscopy 64 (1996) 109-135.

[29] C. Kübel, A. Thust, TrueImage: a software package for focal-series reconstruction in HRTEM, in: E. Weirich, J.L. Labar, X. Zou (Eds.), Nato Science Series E, Springer, 2005, pp. 373-391.

[30] A.G.W. Leslie, Integration of macromolecular diffraction data, Acta Crystallographica D 55 (1999) 1696-1702. 